\title{
FliI Role in Flagellar Assembly of Salmonella $\Delta$ fliI Mutant Strain Determines Motility and Biofilm Formation
}

\author{
Iram Liaqat ${ }^{1, *}$, Safdar Ali Mirza ${ }^{2}$, Sumera Sajjad $^{3}$, Shaukat Ali ${ }^{1, *}$, \\ Muhammad Faiz Qamar ${ }^{4}$ and Ikram Ul Haq ${ }^{5}$ \\ ${ }^{1}$ Department of Zoology, Govt. College University, Lahore-54000 \\ ${ }^{2}$ Department of Botany, Govt. College University, Lahore-54000 \\ ${ }^{3}$ Department of Zoology, Lahore College for Women University, Lahore \\ ${ }^{4}$ Department of Pathobiology, College of Veterinary and Animal Sciences, \\ Sub-campus Jhang \\ ${ }^{5}$ Institute of Industrial Biotechnology, Govt. College University, Lahore-54000
}

\section{A B S T R A C T}

Biofilms formation is a serious problem in both clinical and environmental settings. Various gram
negative bacteria exhibit biofilm formation mediated by flagellum-mediated motility. Type III protein
secretion systems of several gram-negative bacterial pathogens use flagella to invade foreign surfaces,
host tissues and substrates. Flagellar biosynthesis and function in Salmonella typhimurium is regulated by
$>50$ genes. Bioinformatics analysis of flagellar assembly in $S$. typhimurium identified several conserved
structural elements. In this study, FliI a flagellar protein required for flagellar assembly and involved in
a specialized protein export pathway was cloned and overexpressed. $\Delta$ fliI mutant Salmonella strain was
used to transform fliI overproducing plasmid pTrc99A by electroporation. Using vital dyes (Alexaflour
488 ), visualization of motility was observed in wild type, $\Delta$ fli mutant and fliI complemented strain which
was further assessed by biofilm formation ability. Swimming, swarming motility alongwith significantly
reduced biofilm formation was observed in $\Delta$ fli mutant compared to wild type and fliI complemented
strains. This study will extend initial evidence that FliI plays important role in flagellar export system
and flagellum-mediated rotation is critical for swimming, swarming motility and biofilm formation. The
flagellar basal body has an ancient and evolutionarily conserved macromolecular assembly and known
architecture making it an ideal drug target. The knowledge obtained will help to elucidate mechanism and
design principles necessary to understand protein secretion systems.

Article Information
Received 28 June 2019
Revised 30 July 2019
Accepted 18 August 2020
Available online 13 October 2021
Authors' Contribution
IL conceived and designed the study.
IL and SS performed the experiments
and wrote the article. IL, SAM and
SA, MFQ analyzed the data. IL and
IUH helped in reviewing manuscript.
Key words
FliI, Salmonella, Alexa fluor staining,
AfiI mutant, Flagellar motility,
Complementation study.

\section{INTRODUCTION}

$\mathrm{M}$ otility and biofilm formation plays important role in bacterial pathogenesis hence making this aspect ideal to understand bacterial physiology. Almost every microbe including Pseudomonas aeruginosa, Vibrio cholerae, Salmonella and pathogenic E. coli, has ability to show motility but how this motility contribute to biofilm forming ability has not thoroughly studied (Chevance and Hughes, 2008).

Bacteria exhibit both swimming and surface swarming. In aqueous medium, microbes prefer swimming motility while on semi-solid surfaces, swarming, collective migration of bacteria occurs (Fraser and Hughes, 1999). For swarming motility, vegetative bacteria undergo a process of elongation and hyper flagellation which make

\footnotetext{
Corresponding author: iramliaq@hotmail.com 0030-9923/2021/0006-2381 \$9.00/0

Copyright 2021 Zoological Society of Pakistan
}

them highly virulent (Harshey et al., 1994). Furthermore, in this mode bacteria also have the increased ability to form a biofilm thus enhancing their pathogenesis ability (Kearns, 2010; Murray et al., 2010). A biofilm means bacteria adhered to surface and encased in self-secreted exopolysaccharide (EPS) matrix (Branda et al., 2005; Hall-Stoodley and Stoodley, 2009). Biofilm formation enables bacteria to survive well in host by inactivating both innate and adaptive immune responses.

The flagellar contribution to pathogenicity has been studied in many bacteria including Helicobacter pylori, Campylobacter sp., Legionella sp., Aeromonas sp. and Vibrio sp. (Bigot et al., 2005). In a recent study by Chakroun et al. (2018), authors investigated the role of flagella in virulence and biofilm formation. Salmonella typhimurium uses variety of virulence factors, including flagella, fimbriae, adhesins, and invasins to exhibit motility leading to biofilm formation. The flagellar assembly requires approximately 50 genes (Chevance and Hughes, 2008). Structural and other proteins required for export are transported through a flagellar mediated type III 
secretion system (T3SS). This system contains six integral membrane proteins: FlhA, FlhB, FliO, FliP, FliQ, FliR (for Salmonellae and other species) at least. Among these flagellar proteins, FliI is the only established ATPase. It interacts with FliJ (no known function), and with a dimer of FliH (an inhibitor of FliI). ATP hydrolysis caused by FliI is important factor in gate-activation process. This suggested that FliI plays important role in energy provision to T3SS (Minamino et al., 2014).

Besides swimming motility, $S$. typhimurium is among earliest serovars to show morphological differentiation of swarmer cells (Harshey et al., 1994). Kim and Surette (2005) studied swarming motility in $S$. typhimurium and linked it to evolutionarily conserved behaviour in Salmonella. Therefore, in this study, we have looked into both swimming and swarming motility of fli complemented S. typhimurium to check if surface swarming exist in S. typhimurium. Furthermore, we investigated the importance of flagellar mediated biofilm formation in perspective to FliI complementation in SJW2702 ( $\Delta$ fiiI) strain. We constructed fli I complemented strains by overexpressing FliI using pTrc99A vector and showed that FliI deleted SJW2702 ( $\Delta$ fil $)$ ) strains are inefficient in energy coupling mechanism of flagellar type III protein export system making them aflagellated. To our knowledge this study is first of its type to demonstrate the role of fli gene in flagellation and biofilm formation

\section{MATERIALS AND METHODS}

Salmonella strains and culture conditions

Bacterial strains Salmonella enterica serovar Typhimurium SJW1103 and SJW2702 ( $\Delta$ FliI) were used in this study. These were already available in lab obtained from Yamaguchi et al. (1986) and Kubori et al. (1992). The bacteria were routinely cultured in Luria-Bertani (LB) broth and agar at $37^{\circ} \mathrm{C}$. When necessary, chloramphenicol $\left(50 \mu \mathrm{gml}^{-1}\right)$ was supplemented to the growth medium.

\section{FliI cloning and complementation}

To construct fiI complemented Salmonella strains, fliI coding regions were amplified from an existing clone of Salmonela typhimurium by performing Pfu PCR using fliI 5'NdeI, AAAAAACATATGGAGTGCTCTGAATGACCAC and fli 3'EcoR1, AAAAAAGAATTGCTTTGAGTGTTTCCAGAGC (designed in this study). The resulting $1.4 \mathrm{~kb}$ product was digested with $N d e I$ and $E c o R I$ and ligated into pET-28a $(+)$ plasmid, encoding a His-tagged. The NdeI-EcoRI were cloned into pTrc99A having trc promoter and transformed into E. coli BL21. Insertions of the fliI gene was confirmed by colony PCR, restriction digestion and
DNA sequencing (BigDye v3.1, 3130 Genetic Analyzer; Applied Biosystems). S. typhimurium was transformed by electroporation (E. coli Pulser, Bio-Rad). fli I overproducing plasmid was used to transform FliI deletion mutant Salmonella strain SJW2702 ( $\triangle$ fiil) for complementation.

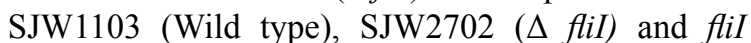
complemented strains were grown and induced with $2 \mathrm{mM}$ isopropyl $\beta$-d-thiogalactoside (IPTG). Cell density was normalized and proteins were precipitated by $10 \%$ trichloroacetic acid (TCA), suspended in a tris-SDS loading buffer and coomassie brilliant blue (CBB) staining was performed.

\section{Growth analysis}

Culture samples were withdrawn at regular intervals to measure the optical density $\left(\mathrm{OD}_{600}\right)$. Replicate growth curve data from the SJW1103 (wild type), SJW2702 ( $\Delta$ fliI) and $f i I$ complemented strains were analyzed by drawing a logarithmic scale through the exponential-growth data points for each experiment (Riaz et al., 2018). Slope was used to calculate the specific growth rate constant.

\section{Motility assay}

Sixteen $\mathrm{h}$ old wild type and fiI Salmonella cultures were grown in motility medium supplemented with $0.1 \%$ glycerol and chloramphenicol $\left(50 \mu \mathrm{gml}^{-1}\right)$. Swimming motility was observed by incubating plates for $5 \mathrm{~h}$ without being inverted. For swarming motility, a region extending $\sim 1 \mathrm{~cm}$ into the colony was observed by phase contrast microscopy following Turner et al. (2010).

\section{Fluorescent staining of flagella}

Fluorescent staining of flagella was performed by following protocol by Turner et al. (2010). Briefly, swarm cells were collected, washed and centrifuged. Pellet was gently suspended in motility medium and thiol-reactive dye (Alexa Fluor 488; Invitrogen-Molecular Probes) was added. Cells were washed with motility medium and Image $J$ was used to measure the lengths of the cell bodies and numbers of flagella (http://rsb.info.nih.gov/ij/). This information was used to measure the polymorphic transition in flagella following Calladine (1975).

\section{Biofilm formation analysis}

Biofilm assay was performed as described previously by Liaqat et al. (2016). This was done in two stages. In first stage, time kinetics for biofilm formation by all three strains was performed following Liaqat and Sakellaris (2012). Second stage of biofilm formation was performed using two assays. In test tube essay, LB medium supplemented with antibiotic was prepared and inoculated and incubated for $96 \mathrm{~h}$. In air-liquid interface method, 
nutrient broth solution was inoculated and poured in petri plates. Coverslips were very cautiously placed aseptically followed by measurement of optical density $\left(\mathrm{OD}_{595}\right)$. Both test tubes and air liquid interface coverslip assays were performed two times for all Salmonella strains, and the averages and standard deviations were calculated for all repetitions of the experiment.

\section{Statistical analysis}

Statistical analyses were performed using student "t-test" for independent samples. All the experiments were performed three times. Data was analysed using Microsoft Excel and SPSS 18. The level of significance was $\mathrm{P}<0.05$.

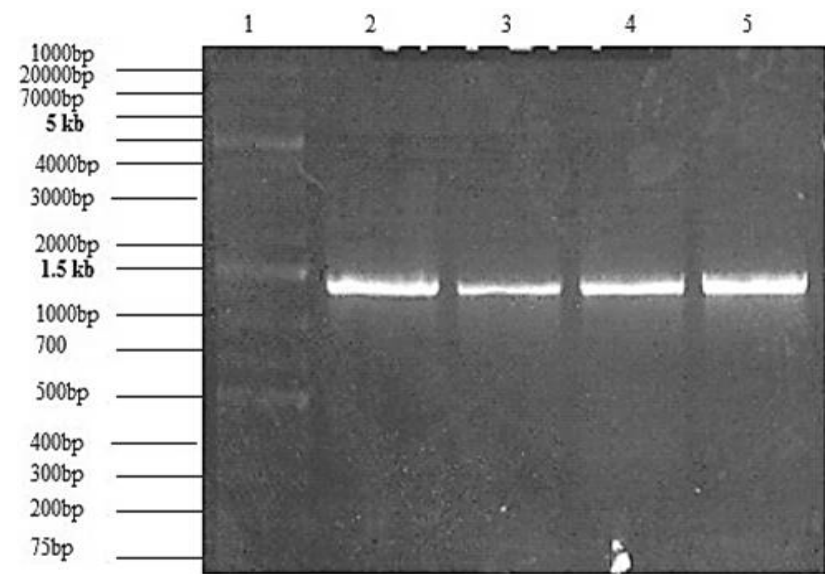

Fig. 1. Agarose gel electropherograms of the $f i I$ from wild type and fiI complemented Salmonella using phusion PCR. $1^{\text {st }}$ Lane, $1 \mathrm{~kb}$ DNA ladder plus PageRuler ${ }^{\mathrm{TM}}$ Prestained Protein Ladder; $2^{\text {nd }}$ Lane, fli (wild type); $3^{\text {rd }}-5^{\text {th }}$ Lane, fli from fiI complemented Salmonella at different annealing temperatures of 60,62 and $65^{\circ} \mathrm{C}$.

\section{RESULTS}

\section{FliI cloning and SDS-PAGE analysis of Salmonella typhimurium}

To verify the role of FliI, we constructed fiI complemented strains. Figure 1 shows $1.4 \mathrm{~kb}$ band of interest on the agarose gel electrophorses using phusion PCR amplification. This fragment encoding fliI was subcloned into pET-28a $(+)$ vector. Subsequent restriction digestion and sequencing verified the recombinant plasmid. The double enzyme digestion of the recombinant plasmids demonstrated successful ligation into the vectors. Afterwards, sequencing confirmed that the sequence of cloned fragment to be accurate (data not shown).

We introduced fliI overproduction plasmid into Salmonella SJW2702 ( $\Delta$ fil $)$. SDS-PAGE anlaysis of each salmonella strain revealed that the amounts of overexpressed fliI was almost similar in both SWJ2102 (wild type) and fiI complemented strains, as seen in sodium dodecyl sulfate gels (Fig. 2, $2^{\text {nd }}$ and $3^{\text {rd }}$ lane). There was no apparent FliI bands in deletion mutants of Salmonella SJW2702 ( $\Delta$ fliI) (Fig. 2, $1^{\text {st }}$ lane), fliI bands were seen in wild type even in the absence of IPTG. In the presence of $2 \mathrm{mM}$ IPTG, fiI bands was the major ones in the whole-cell extracts (Fig. 2, $4^{\text {th }}$ lane).

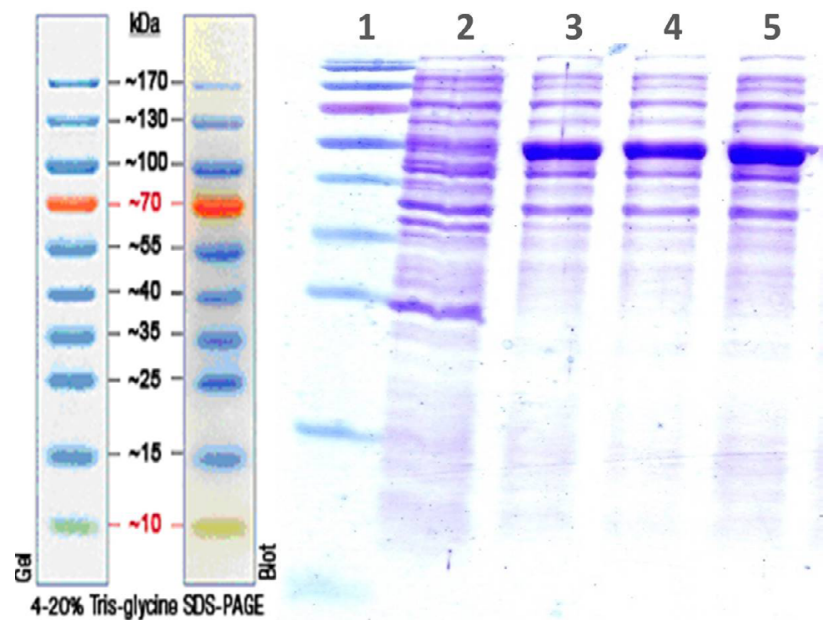

Fig. 2. Sodium dodecyl sulfate-polyacrylamide electrophoresis pattern of FliI in whole-cell extracts from SJW2702, SWJ1102 (wild type Salmonella), and fli complemented Salmonella strains induced with IPTG. $1^{\text {st }}$ Lane, PageRuler ${ }^{\mathrm{TM}}$ Prestained protein ladder; $2^{\text {nd }}$ Lane, without plasmid; $3^{\text {rd }}$ Lane, with plasmid and in the absence of IPTG; $4^{\text {th }}$ and $5^{\text {th }}$ Lanes, with plasmid and in the presence of $2 \mathrm{mM}$ IPTG.

\section{Growth and motility assays}

There was no calculated difference in growth rate of bacteria as was observed by their growth curve and specific growth rate calculations (Fig. 3A, B). Comparison of swimming and swarming motility assays among three strains showed that SJW1102 (wild-type) and fli complemented Salmonella strains produced a large swim ring while the fliI deletion mutant Salmonella SJW2702 stayed at point of inoculation showing no motility (Data not shown). Phase contrast microscopy of fiI complemented cells showed that all cells had flagella (Fig. 4A). Following Calladine (1975), polymorphic transitions were observed in flagellated strains. Most of the flagella in our study were semicoiled in nature (Fig. 4B). To understand the role of fli in swarming motility, we grew bacteria on swarm agar plates containing LB and $0.35 \%$ agar. SJW 1102 and fli complemented strains displayed motility on media. However, SJW2702 ( $\Delta$ fliI) failed to exhibit any swarming motility on $0.35 \%$ agar. 

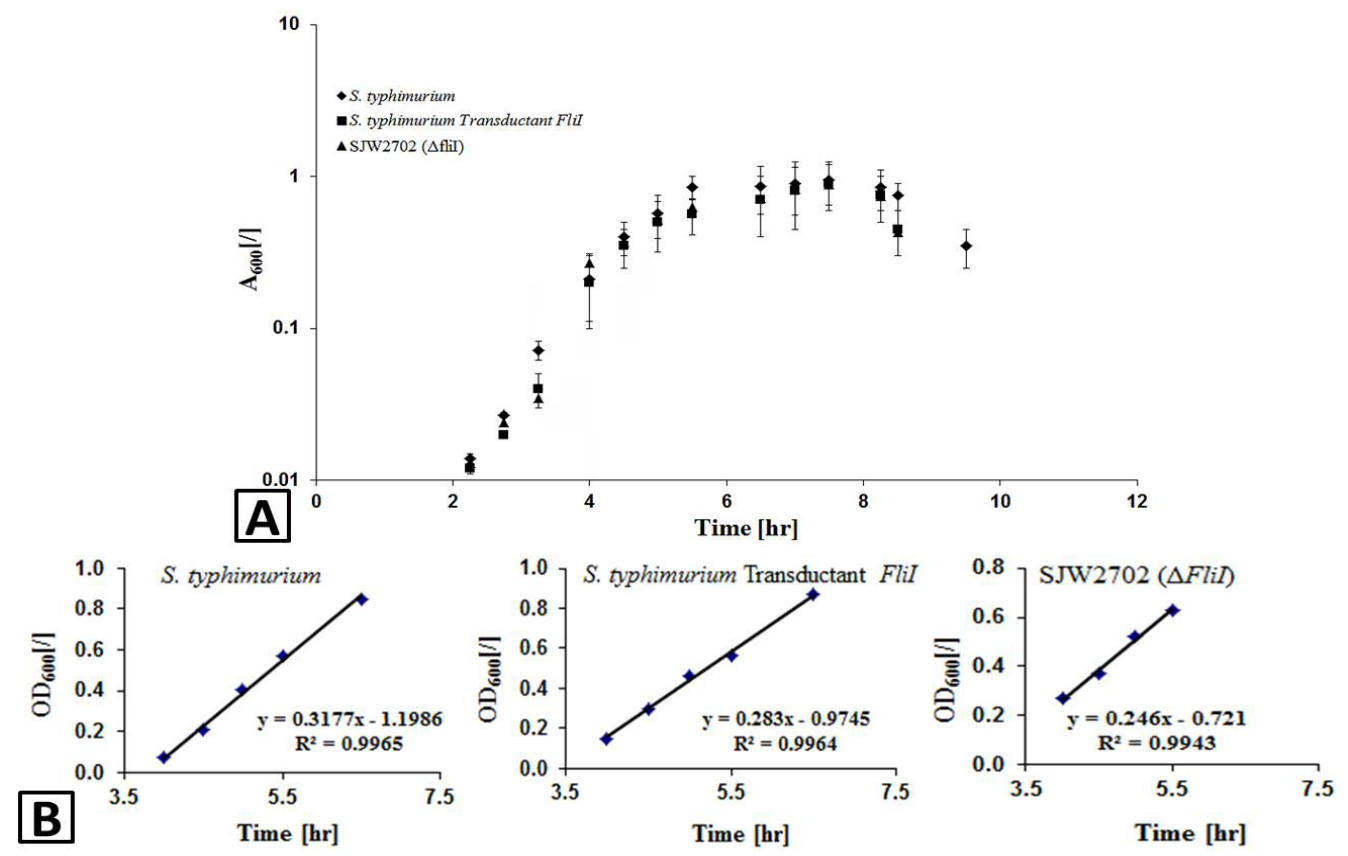

Fig. 3. A, S. typhimurium growth curves. Three strains of Salmonella including wild type, S. typhimurium complemented fiI and SJW2702 ( $\triangle \mathrm{flil}$ ) were grown in Luria-Bertani broth at $37^{\circ} \mathrm{C}$ with aeration. Bacterial growth was determined by $\mathrm{OD}_{600}$. Data were obtained from the average of three independent experiments; B, specific growth rate of three strains.

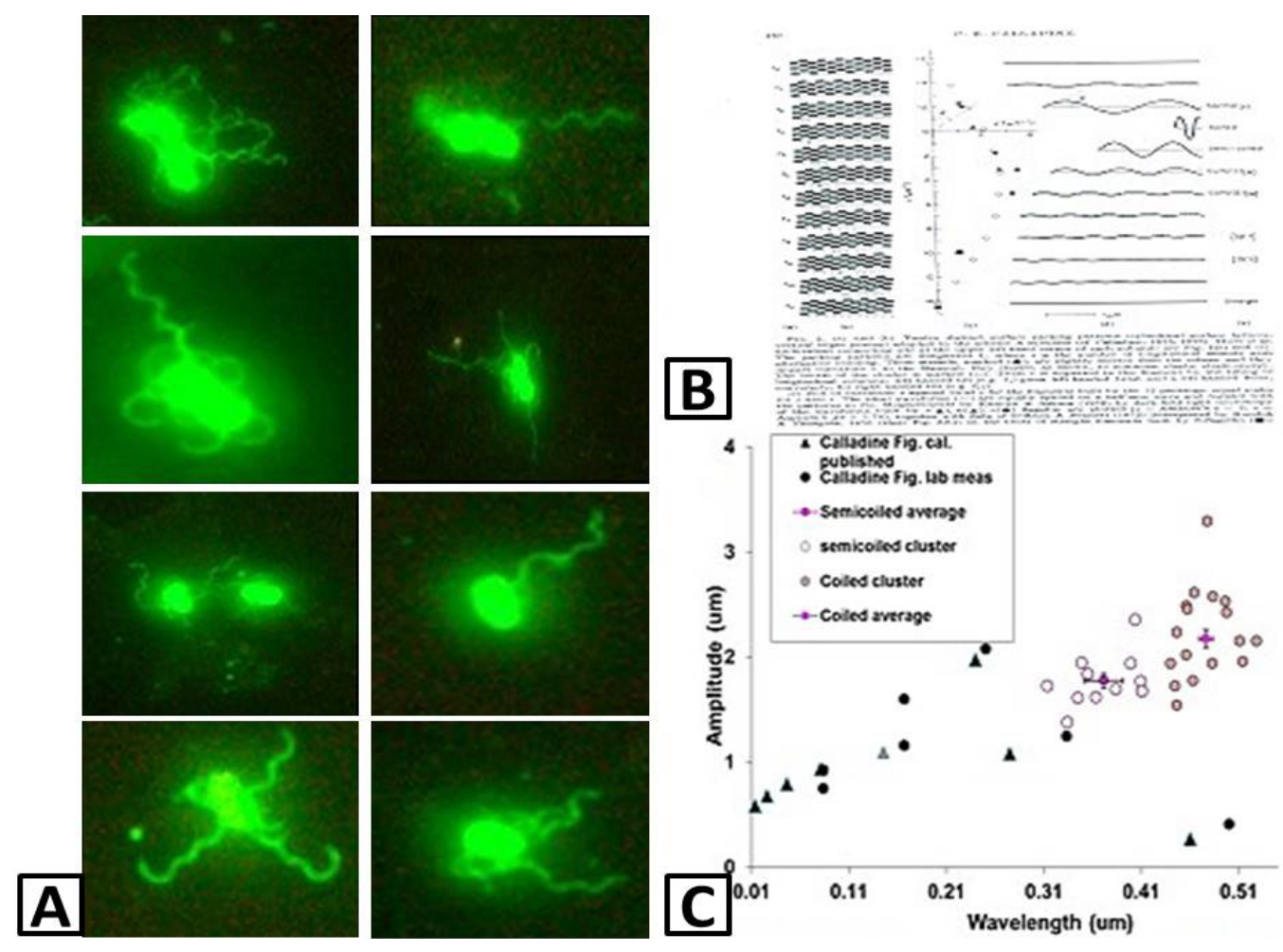

Fig. 4. A, phased contrast microscopy of Alexa flour-488 labeled flagellar strains of fli complemented Salmonella; B, Calladine (1975) model; C, polymorphic transitions were calculated in flagella following Calladine (1975) model and placed in semi-coiled category. 

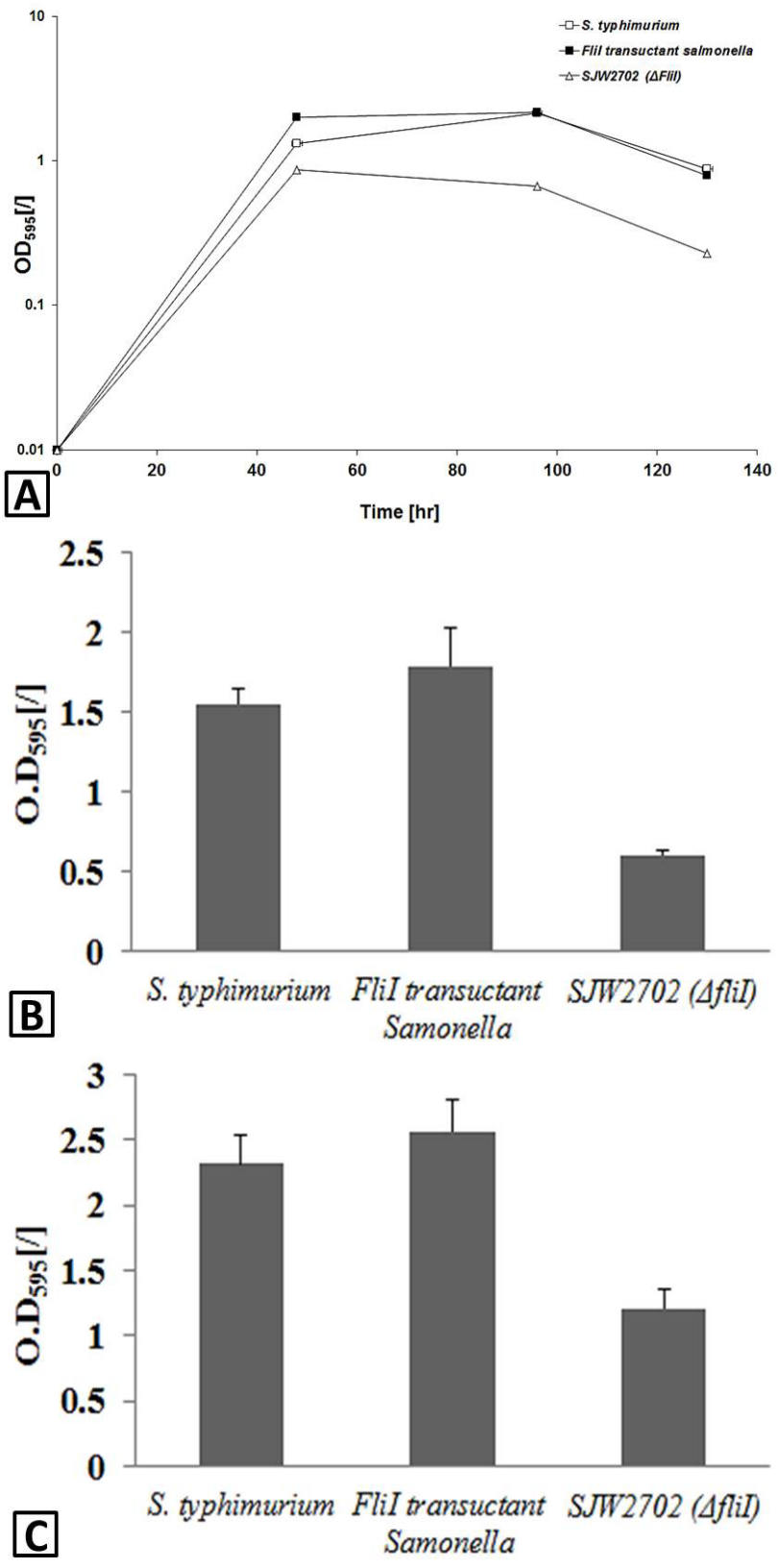

Fig. 5. A, Time kinetics for Biofilm formation. Bacteria were grown for 16 hours and diluted in fresh Luria Bertani medium supplemented with antibiotic. Data was obtained from the average of three independent experiments. B, biofilm formation assays by wild type and fil complemented Salmonella strains by liquid-interface coverslip assay. C, biofilm formation assays by wild type and fil complemented Salmonella strains by test tube method. All three strains were grown in LB medium for $96 \mathrm{~h}$. O.D was measured at $595 \mathrm{~nm}$. fil complemented Salmonella showed best biofilm formation compared to both wild type and fli deleted SWJ2702. Experiment was performed in duplicate.
Biofilm formation study

The biofilm-formation by all three strains of Salmonella was quantified using crystal violet staining method. Results of biofilm time kinetic indicated that all tested Salmonella isolates produced strong biofilm after $96 \mathrm{~h}$. Afterwards, a decline in biofilm formation was observed (Fig. 5A). Once biofilm-forming capacity of different Salmonella isolates was assessed, we investigated further the difference in biofilm forming capacity of all three isolates using test tubes and liquid interface coverslip assays. We were interested to see whether fiI complemented strain has good biofilm forming capacity similar to swimming and swarming motility. As expected, strong biofilm formation by fiI complemented strain was observed compared to both wild type and fiI deleted strains strain (Fig. 5B, C) using both assays.

\section{DISCUSSION}

Flagellar apparatus has been observed to play crucial role in pathogenesis of a great diversity of intestinal pathogens. In this regard, the flagellar assembly of $S$. typhimurium is an interesting aspect to understand bacterial adherence mechanism and biofilm study. There is not much data about translocation of flagellar proteins from the cytosol to the distal end. Among several proteins studied so far, FliI is especially interesting because of its identical nature to catalytic $\beta$ subunit of the $\mathrm{F}_{1}$-ATPase and homology to various proteins in T3SS. Majority of bacteria including both Gram positive and Gram negative have FliI playing ATPase role in type three secretory system (T3SS). In this study, we have analyzed FliI role via complementation in flagellum-mediated rotation for swimming, swarming motility and biofilm formation.

We observed that FliI complemented strains have no difference in growth compared to motility and biofilm formation. Optimum swimming and swarming motility independent of planktonic growth observed in wild type and FliI complemented strains compared to SJW2702 $(\Delta f l i)$ is related to fact that fliI deletion lead to lack of energy for flagellar export. In a previous study, novel motility regulators were screened by genomic analysis and 130 mutations were found to be important to influence motility in S. typhimurium genome (Bogomolnaya et al., 2014). In fact, two energy sources used by flagellar export system include ATP and proton motive force (PMF). FliI forms a homo-hexamer and is the only ATPase of the export system. Although FliI makes export gate highly highly efficient, however, its role is still unclear because of limited information about ATPase mechanistic nature (Minamino et al., 2014). 
It has been documented in several previous studies that either complete flagella or parts of it could promote bacterial adhesion and binding to the host's surfaces thus enhancing virulence. Biofilm formation is an adaptation by different bacterial species to enhance survival and pathogenesis. We observed biofilm formation by all Salmonella isolates in this study. This might be due to greater glycocalyx production at that stage as reported by MacFarlane et al. (2007). However, Salmonella strain lacking fliI gene exhibited almost one fold decrease in biofilm formation compared to wild type and fliI complemented strain. Wood et al. (2006) demonstrated that flagella are important both in biofilm initiation and development. Likewise, Olsen et al. (2013) reported that serovar-specific differences are important in determining in the flagellar involvement as well as chemotaxis genes in attachment and invasion of Salmonella to the host

Following time kinetics of biofilm formation, its quantification was performed using test tubes and liquid interface coverslips assays. Significantly decreased biofilm in fli deficient strains observed in this study might be due to lack of flagella. Non-motile flagellar mutants might have decreased initial surface attachment hence showed poor biofilm formation (Liaqat et al., 2016). Additionally other factors including fimbriae, pili, curli may also contribute to decreased biofilm formation in non flagellar strains (Reisner et al., 2006; Lemon et al., 2007; MacFarlane and Dillon, 2007; Kim et al., 2008; Liaqat and Sakellaris, 2012). Importantly, higher biofilm formation observed by fli complemented strain even compared to wild type. This might be due to the fact that FliI overexpression essentially means more energy production for flagellar export leading to enhanced flagellation hence initiating formation of biofilm. Previous studies by Lemon et al. (2007) and Gorski et al. (2009) are consistent with our finding that without flagella or flagella motility, biofilm formation was significant reduced.

The present study suggested that motility is stringently controlled by an organized flagellar assembly. We concluded that FliI is essential for flagellation, motility and biofilm formation in Salmonella strains. We are the first one to report that without FliI, motility is essentially lost leading to significantly reduced biofilm formation in $S$. typhimurium. More detailed studies on this aspect will lead to better understanding of various mechanisms involved in Salmonella motility at molecular level. Among several questions about the role of flagella, one question which we are interested to address was the role of flagellation in attenuation of strain pathogenesis as was observed by Yang et al. (2012). Efforts will be made in our lab to study fii complemented role through in vivo trails.

\section{ACKNOWLEDGEMENT}

We are grateful to Shahid Khan, Molecular Biology Consortium, Lawrence Berkeley National Laboratory, Berkeley, CA-94720, USA for his criticism and support. Initial phase of this study was financially supported due to his collaboration with LUMS, Lahore, Pakistan.

\section{Statement of conflict of interests}

Authors declare no conflict of interests.

\section{REFERENCES}

Bigot, A., Pagniez, H., Botton, E., Fréhel, C., Dubail, I., Jacquet, C., Charbit, A. and Raynaud, C., 2005. Role of FliF and FliI of Listeria monocytogenes in flagellar assembly and pathogenicity. Infect. Immun., 73: 5530-5539. https://doi.org/10.1128/ IAI.73.9.5530-5539.2005

Bogomolnaya, L.M., Aldrich, L., Ragoza, Y., Talamantes, M., Andrews, K.D., McClelland, M. and Andrews-Polymenis, H.L., 2014. Identification of novel factors involved in modulating motility of Salmonella enterica serotype Typhimurium. PLoS One, 9: e111513. https://doi.org/10.1371/journal. pone. 0111513

Branda, S.S., Vik, S., Friedman, L. and Kolter, R., 2005. Biofilms: The matrix revisited Trends. Microbiology, 13: 20-26. https://doi.org/10.1016/j. tim.2004.11.006

Calladine, C.R., 1975. Construction of bacterial flagella. Nature, 255: 121-124. https://doi. org $/ 10.1038 / 255121 \mathrm{a} 0$

Chakroun, I., Mahdhi, A., Morcillo, P., Cordero, H., Cuesta, A., Bakhrouf, A., Mahdouani, K. and Esteban, M.Á., 2018. Motility, biofilm formation, apoptotic effect and virulence gene expression of atypical Salmonella Typhimurium outside and inside Caco-2 cells. Microb. Pathog., 114: 153-162. https://doi.org/10.1016/j.micpath.2017.11.010

Chevance, F.F. and Hughes, K.T., 2008. Coordinating assembly of a bacterial macromolecular machine. Nat. Rev. Microbiol., 6: 455-465. https://doi. org/10.1038/nrmicro1887

Fraser, G.M. and Hughes, C., 1999. Swarming motility. Curr. Opin. Microbiol., 2: 630-635. https://doi. org/10.1016/S1369-5274(99)00033-8

Gorski, L., Duhé, J.M. and Flaherty, D., 2009. The use of flagella and motility for plant colonization and fitness by different strains of the foodborne pathogen Listeria monocytogenes. PLoS One, 4: e5142. https://doi.org/10.1371/journal.pone.0005142 
Hall-Stoodley, L. and Stoodley, P., 2009. Evolving concepts in biofilm infections. Cell. Microbiol., 11: 1034-1043. https://doi.org/10.1111/j.14625822.2009.01323.x

Harshey, R.M. and Matsuyama, T., 1994. Dimorphic transition in Escherichia coli and Salmonella Typhimurium: surface-induced differentiation into hyperflagellate swarmer cells. Proc. natl. Acad. Sci. USA, 91: 8631-8635. https://doi.org/10.1073/ pnas.91.18.8631

Kearns, D.B., 2010. A field guide to bacterial swarming motility. Nat. Rev. Microbiol., 8: 634-644. https:// doi.org/10.1038/nrmicro2405

Kim, T.J., Young, B.M. and Yong, G.M., 2008. Effect of flagellar mutations on Yersinia enterocolitica biofilm formation. Appl. environ. Microbiol., 74: 5466-5474. https://doi.org/10.1128/AEM.0022208

Kim, W. and Surette, M.G., 2005. Prevalence of surface swarming behavior in Salmonella. J. Bact., 187: 6580-6583. https://doi.org/10.1128/ JB.187.18.6580-6583.2005

Kubori, T., Shimamoto, N., Yamaguchi, S., Namba, K. and Aizawa, S.I., 1992. Morphological pathway of flagellar assembly in Salmonella typhimurium. J. mol. Biol., 226: 433-446. https:// doi.org/10.1016/0022-2836(92)90958-M

Lemon, K.P., Higgins, D.E. and Kolter, R., 2007. Flagellar motility is critical for Listeria monocytogenes biofilm formation. J. Bact., 189: 4418-4424. https://doi.org/10.1128/JB.01967-06

Liaqat, I. and Sakellaris, H., 2012. Biofilm formation and binding specificities of CFA/I, CFA/II and CS2 adhesions of enterotoxigenic Escherichia coli and Cfae-R181A mutant. Braz. J. Microbiol., 43: $\quad 969-980$. https://doi.org/10.1590/S151783822012000300018

Liaqat, I., Sumbal, F. and Sabri, A.N., 2009. Tetracycline and chloramphenicol efficiency against selected biofilm forming bacteria. Curr. Microbiol., 59: 212220. https://doi.org/10.1007/s00284-009-9424-9

Liaqat, I., Pervaiz, Q., Bukhsh, S.J. and Jahan, N., 2016. Investigation of bactericidal effects of medicinal plant extracts on clinical isolates and monitoring their biofilm forming potential. Pak. Vet. J., 36: 159-164.

MacFarlane, S. and Dillon, J.F., 2007. Microbial biofilms in the human gastrointestinal tract. $J$. appl. Microbiol., 102: 1187-1196. https://doi. org/10.1111/j.1365-2672.2007.03287.x
Minamino, T., Morimoto, Y.V., Kinoshita, M., Aldridge, P.D. and Namba, K., 2014. The bacterial flagellar protein export apparatus processively transports flagellar proteins even with extremely infrequent ATP hydrolysis. Scient. Rep., 22: 7579. https://doi. org/10.1038/srep07579

Murray, T.S., Ledizet, M. and Kazmierczak, B.I., 2010. Swarming motility, secretion of type 3 effectors and biofilm formation phenotypes exhibited within a large cohort of Pseudomonas aeruginosa clinical isolates. J. med. Microbiol., 59: 511-520. https:// doi.org/10.1099/jmm.0.017715-0

Olsen, J.E., Hoegh-Andersen, K.H., Casadesus, J., Rosenkranzt, J., Chadfield, M.S. and Thomsen, L.E., 2013. The role of flagella and chemotaxis genes in host pathogen interaction of the host adapted Salmonella entericaserovar Dublin compared to the broad host range serovar S. Typhimurium. BMC Microbiol., 13:67. https://doi: 10.1186/1471-218013-67

Reisner, A., Krogfelt, K.A., Klein, B.M., Zechner, E.L. and Molin, S., 2006. In vitro biofilm formation of commensal and pathogenic Escherichia coli strains: Impact of environmental and genetic factors. $J$. Bact., 188: 3572-3581. https://doi.org/10.1128/ JB.188.10.3572-3581.2006

Riaz, A., Noureen, S., Qamar, M.F., Liaqat, I., Arshad, M. and Arshad, N., 2018. Characterization of bacteriocin like inhibitory substances from Enterococcus ratti MF183967. Pak. Vet. J ., 39:1-6.

Turner, L., Zhang, R., Darnton, N.C. and Berg, H.C., 2010. Visualization of Flagella during bacterial Swarming. J. Bact., 192: 3259-3267. https://doi. org/10.1128/JB.00083-10

Wood, T.K., González Barrios, A.F., Herzberg, M. and Lee, J., 2006. Motility influences biofilm architecture in Escherichia coli. App. Microbial. Cell Physiol., 72: 361-367. https://doi.org/10.1007/ s00253-005-0263-8

Yamaguchi, S., Aizawa, S.I., Kihara, M., Isomura, M., Jones, C.J. and Macnab, R.M., 1986. Genetic evidence for a switching and energy-transducing complex in the flagellar motor of Salmonella typhimurium. J. Bact., 168: 1172-1179. https://doi. org/10.1128/JB.168.3.1172-1179.1986

Yang, X., Thornburg, T., Suo, Z., Jun, S., Robison, A., Li, J., Lim, T., Cao, L., Hoyt, T., Avci, R. and Pascual, D.W., 2012. Flagella overexpression attenuates Salmonella pathogenesis PLoS One, 7: e46828. https://doi.org/10.1371/journal.pone.0046828 